\title{
The impact of tumor size change after target therapy on survival: analysis of patients enrolled onto three clinical trials of advanced NSCLC from one institution
}

\author{
This article was published in the following Dove Press journal: \\ OncoTargets and Therapy \\ 14 November 2012 \\ Number of times this article has been viewed
}

\author{
Jianwei Zhang* \\ Yan Huang* \\ Xiaoling Li \\ Ying Guo \\ Yuanyuan Zhao \\ Cong Xue \\ Zhihuang $\mathrm{Hu}$ \\ Li Zhang \\ Hongyun Zhao
}

State Key Laboratory of Oncology in South China, Sun Yat-Sen University

Cancer Center, Guangzhou, China

*These authors contributed equally to this work and share the first authorship
Correspondence: Li Zhang State Key Laboratory of Oncology in South China, Department of Medical Oncology, Sun Yat-Sen University Cancer Center, 65I Dongfeng Road East, Guangzhou, Guangdong, 510060 , PR China

Tel +86 2087343458

Fax +86 2087343392

Email zhangli6@mail.sysu.edu.cn

Hongyun Zhao

State Key Laboratory of Oncology in South China, Sun Yat-Sen University Cancer Center, 65I Dongfeng Road East, Guangzhou, Guangdong, 510060 , PR China

Tel +86 2087343786

Fax +862087343786

Email zhaohy@sysucc.org.cn
Purpose: To explore whether changes in tumor size impact survival in advanced non-small-cell lung cancer (NSCLC) after target therapy, especially in patients with evaluation of stable disease (SD), and to review the applicability of the Response Evaluation Criteria in Solid Tumors (RECIST) criteria in target therapy.

Patients and methods: Data from 88 NSCLC patients receiving gefitinib (250 mg, daily [qd]), erlotinib (150 mg, qd), and ZD6474 (100 mg, qd) in three clinical trials (IRESSA registration clinical trial, TRUST study, ZD6474 study) during November 2003 to June 2005 were retrospectively analyzed. The treatment effect (complete response, partial response, stable disease [SD], or progressive disease) was evaluated with radiologic assessment according to the RECIST criteria. SD patients were divided into two groups: SD-/0, in which the sum of the longest diameter of target lesions decreased by less than $30 \%$ or did not change; and SD+, in which the sum of the longest diameter of target lesions increased by less than $20 \%$. The differences of progression-free survival (PFS) and overall survival (OS) between these groups were analyzed.

Results: In the whole group, 27 patients achieved complete response or partial response as best response, 40 achieved SD, and 22 had progressive disease. The median PFS and OS were 4 months and 11.1 months, respectively. In SD patients, 27 were SD-/0 and 13 patients were $\mathrm{SD}+$. The PFS and OS of SD+ patients was shorter than that of SD-/0 patients $(5.65$ months vs 2.03 days, $P<0.001$ and 12.2 months vs 7.1 months, $P<0.001$ ).

Conclusion: The applicability of RECIST criteria was called into question in the evaluation of target therapy. Change in tumor size might predict survival in advanced NSCLC patients with target therapy and may be a surrogate endpoint for efficacy in target therapy.

Keywords: non-small-cell lung cancer, target therapy, stable disease, prognostic factor

\section{Introduction}

Lung cancer is the main cause of cancer mortality worldwide, with 1.4 million deaths per year. Non-small-cell lung cancer (NSCLC) accounts for about $85 \%$ of all cases of lung cancer; of these, about $25 \%-30 \%$ of NSCLC patients present with locally advanced disease and $40 \%-50 \%$ present with metastatic disease at initial diagnosis. ${ }^{1,2}$ For decades, platinum-based doublet chemotherapy has been considered as the standard of care for advanced NSCLC patients with a good performance status. Though about $70 \%-80 \%$ of patients experienced clinical benefits after receiving first-line chemotherapy, the overall survival (OS) with "chemotherapy only" remained disappointing, with about 
$15 \%$ of patients surviving 5 years. ${ }^{2}$ Standard cytotoxic chemotherapy reached a "therapeutic plateau," with an OS of $8-10$ months and one year survival rate at $30 \%-35 \%$, according to one study. ${ }^{3}$

In the last decade, with the development of molecular biology and gene research, advances in targeted therapy have provided us with new options that have greatly changed the situation. These agents include the epidermal growth factor receptor-tyrosine kinase inhibitors (EGFR-TKIs), angiogenesis inhibitors, and tumor cell apoptosis inducers., ${ }^{4,5}$ Of these, the EGFR-TKIs, including gefitinib (Iressa ${ }^{\mathrm{TM}}$ [ZD1839]; AstraZeneca, Wilmington, DE) and erlotinib (Tarceva $^{\circledR}$ [OSI-774]; OSI Pharmaceuticals, Melville, NY), are now recommended as first-line treatment for advanced NSCLC patients harboring the EGFR mutation and have greatly improved the survival of this patient population. ${ }^{6}$ Several small molecular tyrosine kinase inhibitors, such as ZD6474, have also been studied in advanced NSCLC, either as single agents or in combination with chemotherapy. ${ }^{7}$

In the early clinical trials, gefitinib, erlotinib and ZD6474 monotherapy were shown to have antitumor activity in the general population with advanced NSCLC. ${ }^{7-9}$ In the era of reliance on cytotoxic drugs as the main anticancer agents, shrinkage of tumor was thought to be a precondition for clinical benefit. Hence, in clinical research of target agents, the main criterion for evaluation of effect was the decrease of tumor size. Currently, the Response Evaluation Criteria in Solid Tumors (RECIST) criteria continue to be used to assess the effect of target therapy. ${ }^{10}$ The RECIST criteria define the measurable and nonmeasurable lesions, the number of target lesions, and the methods of measurement. However, there are some defects in the criteria, and it has been found that the objective response rate of the anticancer drugs has not always been consistent with the clinical benefit. In some kinds of tumors, although the tumor shrunk after treatment with certain agents, the survival benefit was not observed; in the contrast, in other kinds of tumors, although the size of tumor was not changed after treatment, the patients could still attain longer survival. ${ }^{11}$ In fact, some anticancer drugs (especially the target agents) show antitumor effect mainly by slowing down or inhibiting the growth of rather than markedly decreasing the tumor volume, which is different from the traditional cytotoxic chemotherapy. Hence, for these agents, obvious shrinkage of tumor has not been common in the imaging evaluation. In a phase II clinical trial of bevacizumab for metastatic renal cell carcinoma, the response rate was found to be only $10 \%-13 \%{ }^{12}$ But in the phase III clinical trial, it was found that the progression-free survival (PFS) was significantly prolonged in the patients taking bevacizumab (4.8 months vs 2.5 months, $\mathrm{HR}=0.39, P<0.001) .{ }^{13}$ Hence, the disease control rate (complete response or partial response or stable disease), rather than response rate (complete response or partial response), was recommended for the clinical evaluation of a new target agent in research.

From the above, whether the RECIST criteria were suitable for the target therapy assessment has not yet been evaluated. And the impact of absolute tumor size change on survival has not yet been thoroughly studied. Herein, we retrospectively analyzed the advanced NSCLC patients who received target therapy in three clinical trials, to explore the impact of change in tumor size after target therapy on survival in advanced NSCLC patients.

\section{Patients and methods}

\section{General information}

A total of 88 patients with advanced NSCLC who were enrolled in three clinical trials (the IRESSA registration clinical trial, the TRUST study, and the ZD6474 study) of targeted therapy after failure of chemotherapy in Sun Yat-Sen University Cancer Center from December 2003 to October 2007 were retrospectively analyzed. The inclusion criteria: patients age 18 years or older with an Eastern Cooperative Oncology Group (ECOG) performance status between 0-2 were eligible in the presence of documented pathological evidence of advanced (stage IV) NSCLC after failure of one or two prior chemotherapy regimens; enrollees were required to have at least one measurable lesion, adequate hematologic, liver, and renal function, and have a life expectancy of at least 12 weeks. Patients were excluded if they had received systemic treatment, including chemotherapy, target therapy, surgery, or radiotherapy within 4 weeks of study entry. All patients signed informed consent forms that were approved by all the relevant institutional ethical committees, and the process was conducted in accordance with the Declaration of Helsinki and good clinical practice.

Among the 88 patients enrolled, 51 males and 37 females, with median age 55 years (ranging from 26 years to 74 years), the median follow up duration was 12 months. The baseline characteristics of all patients are shown in Table 1.

\section{Treatment}

All the 88 patients were involved in one of three clinical trials. Of the 88, 27 patients received gefitinib $250 \mathrm{mg}$ per day, 42 patients were administered erlotinib $150 \mathrm{mg}$ per day, and 19 patients received ZD6474 $100 \mathrm{mg}$ per day, until objective 
Table I Baseline characteristics and survival of all patients

\begin{tabular}{lll}
\hline Characteristics & Cases $(\mathbf{n}=\mathbf{8 8})$ & Percentage \\
\hline Age (years) & $55(26-74)$ & \\
Median & & \\
Gender & $5 I$ & 57.95 \\
Male & 37 & 42.05 \\
Female & & \\
Smoking status & 46 & 52.27 \\
Never-smoker & 42 & 47.73 \\
Smoker & & \\
Histology & 64 & 72.73 \\
Adenocarcinoma & 24 & 27.27 \\
Nonadenocarcinoma & & \\
Previous chemotherapy & 49 & 55.68 \\
I-2 regimens & 39 & 44.32 \\
$\geq 3$ regimens & & \\
ECOG PS & 81 & \\
0-I & 7 & 29.55 \\
2 & & 45.45 \\
Response & 26 & 25.00 \\
CR+PR & 40 & \\
SD & 22 & \\
PD &
\end{tabular}

Abbreviations: ECOG, Eastern Cooperative Oncology Group; PS, performance status; CR, complete response; PR, partial response; SD, stable disease; PD, progressive disease.

evidence of disease progression, unacceptable toxicity, or withdrawal of consent.

\section{Evaluation}

Target lesion was assessed with computed tomography or magnetic resonance imaging at baseline (within 3 weeks before randomization). The imaging evaluation was performed every 4 weeks in the first 16 weeks and then every 8 weeks. The evaluation of effect was based on RECIST (version 1.0) criteria. Complete response (CR) was defined as the disappearance of all target lesions. Partial response (PR) was defined as at least a $30 \%$ decrease in the sum of diameters of target lesions, taking as reference the baseline sum diameters. Progressive disease (PD) was defined as at least a $20 \%$ increase in the sum of diameters of target lesions, taking as reference the smallest sum on study. Stable disease (SD) referred to a target lesion that was shrinking less than $30 \%$ or that increased less than $20 \%$. The SD population was divided into two groups according to the change in tumor size: SD- 0 , for whom the sum of the longest diameter of target lesions decreased by less than $30 \%$ or did not change, and; SD+, for whom the sum of the longest diameter of target lesions increased by less than $20 \%$.

\section{Statistical analysis}

PFS was defined as the time elapsed from the time of administering target agents to the earliest occurrence of disease progression or death for any reason. OS was defined as the time elapsed from the time of administering target agents to the time of death for any reason. Patients who had not progressed or died at the time of statistical analysis were censored at the time of last follow up. Survival analysis was depicted by the Kaplan-Meier method. Univariate analysis and multivariate analysis were performed with log-rank test and Cox regression analysis, respectively. A $P$ value $<0.05$ was used to denote statistical significance, and all reported $P$ values were two sided. All statistical analyses were performed with SPSS 16.0 (IBM, Armonk, NY).

\section{Results}

All the cases could be evaluated. The objective response rate $(\mathrm{CR}+\mathrm{PR})$ was $29.5 \%$ and the disease control rate $(\mathrm{CR}+\mathrm{PR}+\mathrm{SD})$ was $75 \%$. A total of 26 patients were evaluated as (CR+PR), 40 had a best evaluation of SD, and 22 were evaluated as PD (Table 1).

The median PFS for all the patients was 5.0 months (95\% Confidence Interval [CI], 3.25 6.75) and the median survival time was 13.4 months (95\% CI, 10.92 15.95). Univariate analysis between clinical characteristics and prognosis was performed for all the patients. It was found that in this population, smoking history was the prognostic factor (Table 2). The patients without smoking history showed longer survival time than that of patients with smoking

Table 2 Correlation of basic characteristics and response of the NSCLC patients to the overall survival

\begin{tabular}{|c|c|c|}
\hline Items & $\begin{array}{l}\text { Median survival } \\
\text { (months) }(95 \% \mathrm{Cl})\end{array}$ & $\begin{array}{l}\text { Univariate } \\
\text { analysis } P *\end{array}$ \\
\hline Age (years) & & 0.859 \\
\hline$<60$ & $12.9(8.1-17.7)$ & \\
\hline$\geq 60$ & | $3.4(9.4-17.5)$ & \\
\hline Gender & & 0.225 \\
\hline Male & $11.2(6.8-15.7)$ & \\
\hline Female & $15.0(12.2-17.9)$ & \\
\hline Smoking history & & 0.004 \\
\hline Never-smoker & $15.0(\mid 1.2-18.8)$ & \\
\hline Smoker & $10.6(6.8-14.5)$ & \\
\hline Histology & & 0.215 \\
\hline Adenocarcinoma & $14.6(\mid 2.2-17.0)$ & \\
\hline Nonadenocarcinoma & $8.0(5.0-11.0)$ & \\
\hline Previous chemotherapy & & 0.760 \\
\hline I-2 regimen & $12.9(9.4 \mid-16.40)$ & \\
\hline$\geq 3$ regimens & $14.2(10.7-17.7)$ & \\
\hline ECOG PS & & 0.140 \\
\hline $0-1$ & |3.7 (|0.2-17.2) & \\
\hline 2 & $12.2(1.4-23.0)$ & \\
\hline
\end{tabular}

Note: *Log-rank test.

Abbreviations: NSCLC, non-small-cell lung caner; ECOG, Eastern Cooperative Oncology Group; PS, performance status; CI, Confidence interval. 
history $(P=0.004)$. The correlation between the effect of target therapy and the survival was also observed using univariate analysis. The patients with disease control $(\mathrm{CR}+\mathrm{PR}+\mathrm{SD})$ showed obvious survival benefit when compared with the PD group patients $(P<0.001)$ (Table 3$)$.

The median PFS of the (CR+PR) group, SD group, and PD group was 11.0 months, 5.5 months, and 1.0 month, respectively. And the median survival time was 16.8 months, 15.6 months, and 6.6 months in the three groups respectively. In further comparison, the PFS and OS of both the (CR+PR) group and $\mathrm{SD}$ group were significantly longer than that of the PD group ( $P<0.001$ and $P<0.001$, respectively). But no significant difference was observed between the $(\mathrm{CR}+\mathrm{PR})$ group and SD group in PFS and OS $(P=0.072$ and $P=0.528$, respectively) (Figure 1).

In the 40 patients with the best evaluation of $\mathrm{SD}$, 27 patients had tumor regression (SD-/0 group) and 13 patients had a tumor that slightly enlarged (SD+ group). There was no significant difference between the SD-/0 and $\mathrm{SD}+$ groups with respect to basic characteristics, including smoking history $(P=0.54)$ and pathology type $(P=0.28)$. The group of patients with tumor regression (SD-/0, group) had longer PFS than that of patients with tumor enlargement (SD+ group) (13.7 months vs 2.9 months, $P<0.001$ ), and the OS of the SD-/0 group was also longer than that of SD+ group (18.0 months vs 11.0 months, $P=0.005$ ). Moreover, when compared with the $(\mathrm{CR}+\mathrm{PR})$ group, no significant differences were observed between the SD-/0 group and (CR+PR) group in PFS and $\mathrm{OS}(P=0.794$ and $P=0.676$, respectively). Similarly, the OS of the SD+ group and PD group had no significant difference $(P=0.498)$, though the PFS was longer in SD+ group $(P<0.001)$ (Table 3, Figure 2).
Univariate analysis was performed for the SD group of patients. In this group, both the smoking history and the change of tumor size were found to be related to survival prognosis (Table 3 and Table 4). The patients without smoking history had better prognosis than the patients with smoking history $(P=0.027)$. The multivariate analysis demonstrated that smoking history $(P=0.004)$, disease control $(P<0.001)$, and the tumor size regression $(P=0.027)$ were independent prognostic factors (Table 5).

\section{Discussion}

We retrospectively analyzed the correlation between the efficacy of target therapy and the prognosis in previouslytreated advanced NSCLC. In our research, the response rate $(\mathrm{CR}+\mathrm{PR})$ of all patients was $29.5 \%$, and the disease control rate $(\mathrm{CR}+\mathrm{PR}+\mathrm{SD})$ was $75 \%$. The median survival time was 13.4 months, which was similar to the result of previous reports in unselected NSCLC patients receiving EGFR-TKI. ${ }^{14,15}$ The PFS and OS of the (CR+PR) group and $\mathrm{SD}$ group patients were not significantly different, which indicated that the SD group patients could still obtain survival benefit from target therapy. Interestingly, when analyzing the PFS and OS in the SD group, we found that only the subgroup of patients with tumor regression could obtain survival benefit from target therapy. Longer PFS and OS were observed in SD-/0 group patients when compared with SD+ group. Further, the prognosis of the patients in the SD-/0 group and $(\mathrm{CR}+\mathrm{PR})$ group was found to be similar, and the $\mathrm{SD}+$ group showed similar OS with PD group.

For advanced NSCLC patients receiving platinum-based chemotherapy, it has been reported that the patients with an imaging evaluation of PR had better survival than those with

Table 3 Correlation of different response in NSCLC patients to the overall survival

\begin{tabular}{|c|c|c|c|c|}
\hline Items & $\begin{array}{l}\text { PFS (months) } \\
(95 \% \mathrm{Cl})\end{array}$ & $\begin{array}{l}\text { Univariate } \\
\text { analysis } P^{*}\end{array}$ & $\begin{array}{l}\text { Median survival } \\
\text { (months) }(95 \% \mathrm{Cl})\end{array}$ & $\begin{array}{l}\text { Univariate } \\
\text { analysis } P^{*}\end{array}$ \\
\hline I. $C R+P R+S D$ & $7.0(4.7-9.3)$ & $<0.001$ & $16.3(13.8-18.9)$ & $<0.001$ \\
\hline PD & $1.0(0.9-1.1)$ & & $6.6(4.7-8.4)$ & \\
\hline 2. $C R+P R$ & II.0 (7.9-|4.0) & 0.072 & $16.8(7.9-25.7)$ & 0.528 \\
\hline SD & $5.5(3.9-7.1)$ & & $15.6(12.1-19.1)$ & \\
\hline 3. $P D$ & $1.0(0.9-1.1)$ & $<0.001$ & $6.6(4.7-8.4)$ & $<0.001$ \\
\hline SD & $5.5(3.9-7.1)$ & & $15.6(12.1-19.1)$ & \\
\hline 4. $S D-10$ & $13.7(0.2-27.3)$ & $<0.001$ & $18.0(13.1-22.9)$ & 0.001 \\
\hline $\mathrm{SD}+$ & $2.9(1.8-4.0)$ & & II.0(4.7-17.3) & \\
\hline 5. $S D-10$ & $13.7(0.2-27.3)$ & 0.794 & $18.0(\mid 3.1-22.9)$ & 0.676 \\
\hline$C R+P R$ & II.0 (7.9-14.0) & & $16.8(7.9-25.7)$ & \\
\hline 6. SD & $2.9(1.8-4.0)$ & $<0.001$ & II.0(4.7-17.3) & 0.498 \\
\hline PD & $1.0(0.9-1.1)$ & & $6.6(4.7-8.4)$ & \\
\hline
\end{tabular}

Note: *Log-rank test.

Abbreviations: NSCLC, non-small-cell lung caner; CR, complete response; PR, partial response; SD, stable disease; PD, progressive disease; SD-10, stable disease with tumor regression; SD+, stable disease with tumor enlargement; PFS, progression-free survival; Cl, Confidence interval. 

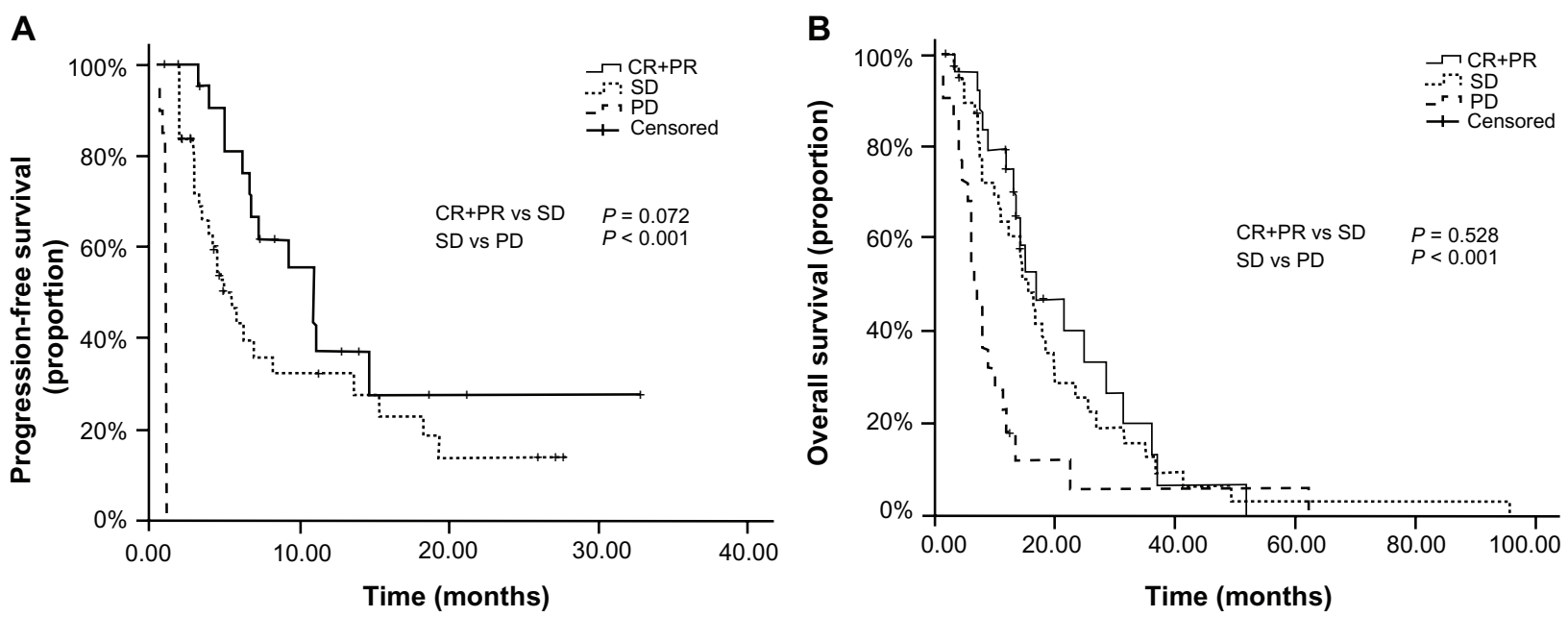

Figure I Progression-free survival and overall survival curves for all patients with different response after target therapy: (A) Comparison of PFS on patients with (CR+PR) vs $S D$ vs $P D ;(B)$ Comparison of $O S$ on patients with $(C R+P R)$ vs $S D$ vs $P D$.

Abbreviations: PFS, progression-free survival; OS, overall survival; CR, complete response; PR, partial response; SD, stable disease; PD, progressive disease.

the evaluation of $\mathrm{SD},{ }^{16}$ which demonstrated the killing-effect characteristics of cytotoxic drugs. However, different from the cytotoxic drugs, the antitumor effect of the target therapy drugs was mainly achieved through inhibiting the growth of tumor. In the clinical trial of sorafenib for renal cancer and hepatocellular carcinoma, ${ }^{12,13}$ the survival benefit was observed in patients with tumor stabilization. Kurata et $\mathrm{al}^{11}$ reported in a meta-analysis that the median survival time of advanced NSCLC patients receiving EGFR-TKI as second-line treatment increased by 0.0375 months with each $1 \%$ increase in stable disease control rate $(P=0.039)$. Yamamoto et a ${ }^{17}$ analyzed a phase III clinical trial and found that the disease control rate $(\mathrm{CR}+\mathrm{PR}+\mathrm{SD})$ was a better predictor of efficacy for target therapy compared with objective response rate (CR+PR). Our results also found that $(\mathrm{CR}+\mathrm{PR})$ group and the $\mathrm{SD}$ group had no significantly different PFS and OS, suggesting that the majority of patients receiving target therapy would have stabilization of tumor after treatment, and that the SD group is worthy of further study. Theoretically, patients in the (CR+PR) group should have better efficacy than patients in the SD group. But it must be remembered that the sample size in our study was relatively small, which might have affected the statistical results. In the 40 patients who had an evaluation of SD, 27 patients had tumor regression (SD-/0 group), and only 13 patients had slight tumor enlargement (SD+ group).
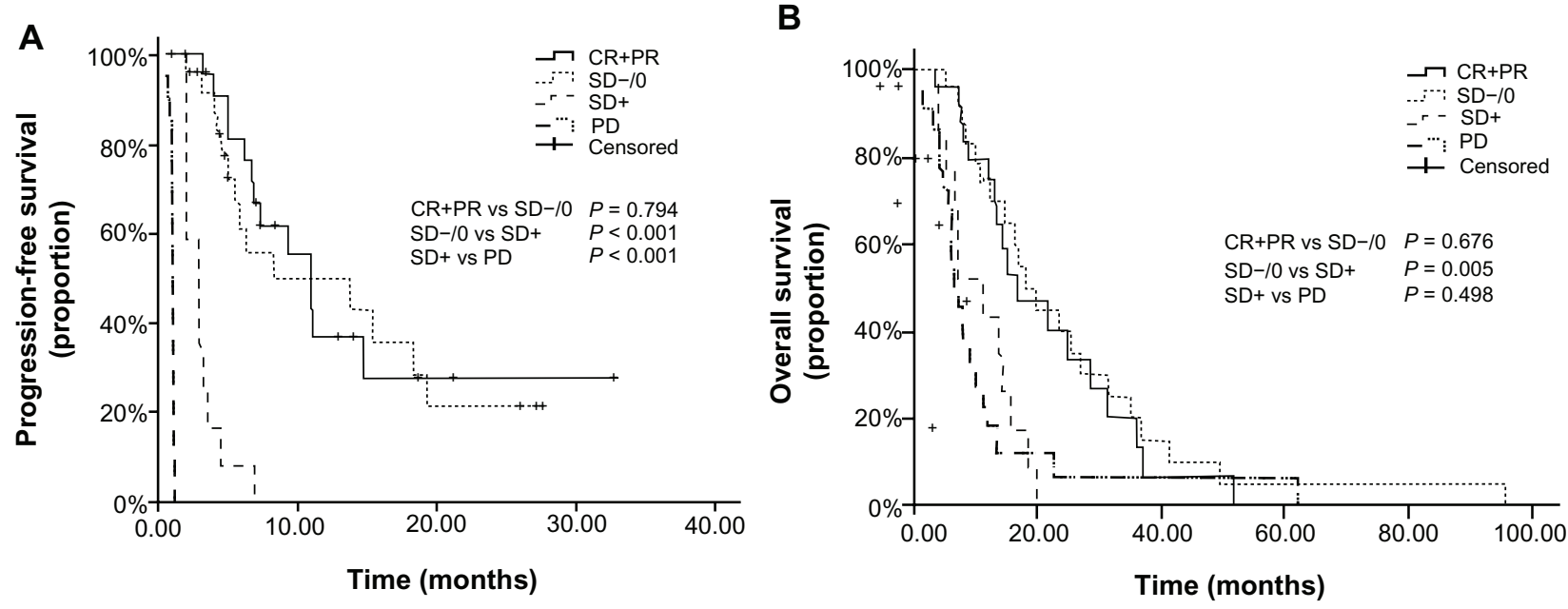

Figure 2 PFS and OS curves after dividing the SD group to SD+ and SD-10 for the patients with different response: (A) Comparison of PFS on patients with (CR+PR) vs SD-10 vs SD+ vs PD; (B) Comparison of OS on patients with (CR+PR) vs SD-10 vs SD+ vs PD.

Abbreviations: PFS, progression-free survival; OS, overall survival; SD, stable disease; SD+, stable disease with tumor enlargement; SD-/0 stable disease with tumor shrinkage or no change; CR, complete response; PR, partial response; PD, progressive disease. 
Table 4 Correlation of basic characteristics in SD patients to the overall survival

\begin{tabular}{|c|c|c|}
\hline Items & $\begin{array}{l}\text { Median survival } \\
\text { (months) }(95 \% \mathrm{Cl})\end{array}$ & $\begin{array}{l}\text { Univariate } \\
\text { analysis } P *\end{array}$ \\
\hline Age (years) & & 0.463 \\
\hline$<60$ & $16.3(10.3-22.4)$ & \\
\hline$\geq 60$ & $14.4(10.2-18.7)$ & \\
\hline Gender & & 0.404 \\
\hline Male & $14.4(10.8-18.0)$ & \\
\hline Female & $16.8(6.0-27.6)$ & \\
\hline Smoking history & & 0.027 \\
\hline Never-smoker & $23.4(8.4-38.4)$ & \\
\hline Smoker & $12.2(7.8-16.6)$ & \\
\hline Histology & & 0.536 \\
\hline Adenocarcinoma & $16.8(12.8-20.8)$ & \\
\hline Nonadenocarcinoma & $10.6(6.2-15.0)$ & \\
\hline Previous chemotherapy & & 0.347 \\
\hline $\mathrm{I}-2$ regimen & $11.0(8.0-14.0)$ & \\
\hline$\geq 3$ regimens & $19.8(8.8-30.8)$ & \\
\hline ECOG PS & & 0.981 \\
\hline $0-1$ & $15.6(\mid 2.4-18.8)$ & \\
\hline 2 & $12.2(12.2-13.4)$ & \\
\hline
\end{tabular}

Note: *Log-rank test.

Abbreviations: SD, stable disease; ECOG, Eastern Cooperative Oncology Group; PS, performance status; $\mathrm{Cl}$, Confidence interval.

The relative small sample size in the $\mathrm{SD}+$ group might have led to the lack of difference in PFS between the CR+PR group and SD group.

However, in this study, we tried to highlight the difference in prognosis between the SD-/0 and SD+ group patients. Not all the SD patients were able to obtain benefit from target therapy. In clinical practice, due to the broad definition of SD according to RECIST criteria, the choices of treatment regimen were disturbing. For patients with tumor enlargement less than $20 \%$ after target therapy, disease progression might occur in a short time under the original treatment regimen (according to the evaluation and clinical guideline), which would lead to treatment delay and the waste of medical resources. On the other hand, in patients with tumor regression less than $30 \%$, indicating that tumor burden was decreasing, termination of treatment too early could deprive them of survival benefit from maintained treatment. To verify a treatment regimen, OS is the most objective parameter in clinical research, but this result is

Table 5 Multivariate analysis

\begin{tabular}{lllll}
\hline Variable & $\begin{array}{l}\text { Adverse } \\
\text { Covariate }\end{array}$ & $\begin{array}{l}\text { Relative } \\
\text { risk }\end{array}$ & $\mathbf{9 5 \%} \mathbf{C l}$ & $\mathbf{P}$ \\
\hline Smoking history & Smoker & 2.50 & $1.48-4.23$ & 0.004 \\
Response & PD & 2.56 & $1.52-4.34$ & $<0.001$ \\
& SD+ & 2.57 & $1.11-5.93$ & 0.027 \\
\hline
\end{tabular}

Abbreviations: $\mathrm{Cl}$, confidence interval; $\mathrm{PD}$, progressive disease; $\mathrm{SD}+$, stable disease with tumor enlargement. obtained too late to guide the choice of regimen in the course of disease; therefore, a more effective surrogate endpoint, with relatively short time intervals, is necessary to monitor the efficacy of the treatment course. Kris ${ }^{18}$ suggested that any increase or decrease of tumor size should be considered as a surrogate endpoint for efficacy. In the present study, we also found that the change of tumor size was correlated with prognosis. Especially in the evaluation of SD group patients, multivariate analysis showed that the change of tumor size was an independent prognostic factor. Patients in the SD-/0 group had significantly better survival than those in the SD+ group. Our results were consistent with those previously reported by Kris, ${ }^{18}$ who analyzed several cytotoxic drugs and target drugs in NSCLC therapy and found that not only the responsive patients could obtain survival benefit, but also those SD patients with tumor regression. These findings indicated that the SD group patients could be divided into different subgroups based on change of tumor size. The patients with tumor size regression had better prognosis. On the contrary, the patients with tumor enlargement in the SD group received little benefit from this kind of target therapy; further, the present study showed that the enlarged-tumor (SD+) group had OS that was similar to the PD group.

Both in clinical practice and clinical trials, the evaluation of effect is very important. The World Health Organization (WHO) criteria were first published in 1981 and assessed tumor burden by summing the products of bidimensional lesion measurement. ${ }^{19}$ However, the number of target lesions, and the measurement method were not defined in the criteria, and it was found that the WHO criteria could lead to confusion in the interpretation of trial results. The RECIST used unidimensional lesion measurement (and defined nonmeasurable lesions) and the number of target lesions, and was a great improvement when compared with the WHO criteria. However, for target therapy with special antitumor characteristics, previous studies ${ }^{16-18}$ and the present study have indicated that the RECIST criteria has certain limitations. In particular, the definition of SD in RECIST criteria was too broad to define the different prognosis groups. This limitation might lead to the wrong assessment in some patients treated with target therapy.

The case number was a limitation of our study. However, the patients in our research were all from three clinical trials of target therapy in advanced NSCLC that were enrolled in one center. The three trials were all multicenter studies. Hence, the case number in only one center was small. All patients were strictly enrolled according to the inclusion criteria, and the imaging evaluation was performed by an 
independent radiologic review committee. The results of this population were therefore more reliably comparable.

To sum up, the applicability of RECIST criteria was doubted in the evaluation of target therapy. Our study found that classification of SD subgroup by tumor size can suitably stratify survival in NSCLC patients treated with target therapy, and may help improve the RECIST criteria and optimize therapy management Moreover, changes of tumor size might be a surrogate endpoint for efficacy evaluation in target therapy. Owing to our limitations of retrospective analysis and small sample size, in the future, further investigation with large sample size is warranted to validate the results.

\section{Acknowledgment}

The work was supported by the Medical Science Foundation of Guangdong Province, China (No A2006309).

\section{Disclosure}

The authors report no conflict of interest in this work.

\section{References}

1. Landis SH, Murray T, Bolden S, Wingo PA. Cancer statistics, 1999. $C A$ Cancer J Clin. 1999;49(1):8-31.

2. Novello S, Le Chevalier T. Chemotherapy for non-small-cell lung cancer. Part 1: Early-stage disease. Oncology (Williston Park). 2003;17(3):357-364.

3. Schiller JH, Harrington D, Belani CP, et al; Eastern Cooperative Oncology Group. Comparison of four chemotherapy regimens for advanced non-small-cell lung cancer. $N$ Engl J Med. 2002;346(2):92-98.

4. Aggarwal C, Somaiah N, Simon G. Antiangiogenic agents in the management of non-small cell lung cancer: where do we stand now and where are we headed? Cancer Biol Ther. 2012;13(5):247-263.

5. Mountzios G, Owonikoko T, Karamouzis M, Argiris A. Current and emerging roles of epidermal growth factor receptor inhibitors in advanced non-small cell lung cancer. The Open Lung Cancer Journal. 2010; 310(57):57-65.

6. Mok TS, Wu YL, Thongprasert S, et al. Gefitinib or carboplatin-paclitaxel in pulmonary adenocarcinoma. $N$ Engl J Med. 2009;361(10):947-957.
7. Sarkar S, Mazumdar A, Dash R, Sakar D, Fisher PB, Mandal M. ZD6474, a dual tyrosine kinase inhibitor of EGFR and VEGFR-2, inhibits MAPK/ERK and AKT/PI3-K and induces apoptosis in breast cancer cells. Cancer Biol Ther. 2010;9(8):592-603.

8. Miller VA, Johnson DH, Krug LM, et al. Pilot trial of the epidermal growth factor receptor tyrosine kinase inhibitor gefitinib plus carboplatin and paclitaxel in patients with stage IIIB or IV non-small-cell lung cancer. J Clin Oncol. 2003;21(11):2094-2100.

9. Shepherd FA, Rodrigues PJ, Ciuleanu T, et al. Erlotinib in previously treated non-small-cell lung cancer. $N$ Engl $J$ Med. 2005;353(2): 123-132.

10. Eisenhauer EA, Therasse P, Bogaerts J, et al. New response evaluation criteria in solid tumours: revised RECIST guideline (version 1.1). Eur J Cancer. 2009;45(2):228-247.

11. Kurata $\mathrm{T}$, Matsuo K, Takada M, et al. Is the importance of achieving stable disease different between epidermal growth factor receptor tyrosine kinase inhibitors and cytotoxic agents in the second-line setting for advanced non-small cell lung cancer? J Thorac Oncol. 2006;1(7):684-691.

12. Escudier B, Eisen T, Stadler WM, et al; TARGET Study Group. Sorafenib in advanced clear-cell renal-cell carcinoma. $N$ Engl $J$ Med. 2007;356(2):125-134.

13. Sandler A, Gray R, Perry MC, et al. Paclitaxel-carboplatin alone or with bevacizumab for non-small-cell lung cancer. $N$ Engl J Med. 2006;355(24):2542-2550.

14. Zhao YY, Zhang Y, Zhao HY, et al. Predictive factors for response and survival of gefitinib-treated locally advanced or metastatic non-small cell lung cancer patients: a retrospective analysis of two phase II clinical trials. CJC. 2009;28(6):626-631. Chinese.

15. Fukuoka M, Yano S, Giaccone G, et al. Multi-institutional randomized phase II trial of gefitinib for previously treated patients with advanced non-small-cell lung cancer (The IDEAL 1 trial). J Clin Oncol. 2003;21(12):2237-2246.

16. Sirohi B, Ashley S, Norton A, et al. Early response to platinum-based first-line chemotherapy in non-small cell lung cancer may predict survival. J Thorac Oncol. 2007;2(8):735-740.

17. Yamamoto N, Nishiwaki Y, Negoro S, et al. Disease control as a predictor of survival with gefitinib and docetaxel in a phase III study (V-15-32) in advanced non-small cell lung cancer patients. J Thoracic Oncology. 2010;5(7):1042-1047.

18. Kris MG. Foreword; challenging the 'one-size-fits-all' approach of chemotherapy and radiotherapy for patients with non-small cell lung cancer. Lung Cancer. 2008;60 Suppl 2:S1-S2.

19. World Health Organization. WHO Handbook for Reporting Results of Cancer Treatment. Offset Publication No 48. Geneva: World Health Organization; 1979. Available from: http://whqlibdoc.who.int/offset/ WHO_OFFSET_48.pdf. Accessed October 18, 2012.
OncoTargets and Therapy

\section{Publish your work in this journal}

OncoTargets and Therapy is an international, peer-reviewed, open access journal focusing on the pathological basis of all cancers, potential targets for therapy and treatment protocols employed to improve the management of cancer patients. The journal also focuses on the impact of management programs and new therapeutic agents and protocols on

\section{Dovepress}

patient perspectives such as quality of life, adherence and satisfaction. The manuscript management system is completely online and includes a very quick and fair peer-review system, which is all easy to use. Visit http://www.dovepress.com/testimonials.php to read real quotes from published authors. 